\title{
Structure and Dynamics of Nondilute Polyfluorene Solutions
}

\author{
G. Fytas, ${ }^{*, t, \neq}$ H. G. Nothofer, ${ }^{\ddagger}$ U. Scherf, ${ }^{\S}$ D. Vlassopoulos, ${ }^{\dagger}$ and G. Meier, ${ }^{\ddagger}$, \\ FO.R.T.H., Institute of Electronic Structure and Laser, P.O. Box 1527, Heraklion, 71110 Crete, Greece; \\ Max-Planck Institut für Polymerforschung, P.O. Box 3148, 55021 Mainz, Germany; Institut für \\ Chemie, Universität Postdam, Karl-Li iebknecht-Str. 24/ 25, D-14476 Golm, Germany; and \\ Forschungszentrum J ülich, Institut für Festkörperforschung, P.O. Box 1913, 52425 J ülich, Germany \\ Received August 6, 2001; Revised Manuscript Received October 16, 2001
}

\begin{abstract}
The rotational and translational diffusion dynamics of newly synthesized polyfluorenes were studied by dynamic light scattering over a broad concentration range up to the lyotropic state. On the basis of small-angle neutron scattering and translational diffusion measurements in dilute solutions, the single polymers were described as wormlike chains with a low persistent length I $=7 \pm 0.5 \mathrm{~nm}$ and cross-section diameter $\mathrm{d}=1.8 \pm 0.5 \mathrm{~nm}$. In the semidilute concentration regime, the rate of increase of the cooperative diffusion and the osmotic pressure with $c / c^{*}$ ( $c^{*}$ being the overlap concentration) was lower than for linear flexible chains in good solvent. The intermediate scattering function displayed a second process assigned to chain relaxation, in reasonable agreement with the longest relaxation time obtained from shear rheometry. The bimodal shape of the orientation correlation function, which is a characteristic feature of shape persistent polymers and exhibits a peculiar dependence on the scattering angle, was also observed in this model system. The fuzzy cylinder model adequately represented the concentration dependence of the osmotic modulus and the chain orientation time, conforming to the wormlike nature of these polymers.
\end{abstract}

\section{Introduction}

The understanding of the average conformation and dynamics of flexible linear polymers in dilute and nondilute solutions is far much more comprehensive compared to their stiff counterparts. ${ }^{1}$ This arises in part from the limited experimental work due to the very few well-characterized semiflexible polymers which gain their rigidity from shape persistence ${ }^{2}$ and not helical structures. ${ }^{1,3}$ The latter polymers usually lack inherent optical anisotropy, a prerequisite to utilize the powerful depolarized light scattering technique, which provides access to rotational motion, a necessary property for the complete understanding of interacting persistent polymers.

Recently, the orientation relaxation function $\mathrm{C}_{\text {or }}(\mathrm{q}, \mathrm{t})$ of poly(p-phenylene) hairy rod polymers ${ }^{2}$ was found to exhibit an unexpected bimodal shape with peculiar dependence on the scattering wavevector $q$, whereas on the other hand the cooperative diffusion was described by renormalization theory. The latter was the main relaxation mechanism of the intermediate scattering function $C(q, t)$ for the concentration fluctuations in semidilute solutions. So far, the contribution of the chain relaxation to the $C(q, t)$ due to the coupling between concentration fluctuations and el astic stress is not fully understood, despite theoretical ${ }^{4}$ and experimental ${ }^{5}$ progress, primarily with flexible polymers. Further, polydispersity and bulky side groups are factors that could affect the rotational dynamics.

In view of the above unresolved issues, to shade more light into the complex dynamics of wormlike shape persistent polymers, we utilized in this work four fractions of a newly synthesized 6 polyfluorene (PF) (Chart 1) with the optical anisotropy lying mainly on

\footnotetext{
$\dagger$ Institute of Electronic Structure and Laser.

‡ Max-Planck Institut für Polymerforschung.

$\S$ Universität Postdam.

$\perp$ Institut für F estkörperforschung.
}

Chart 1. Molecular Structure of the Polyfluorene Used

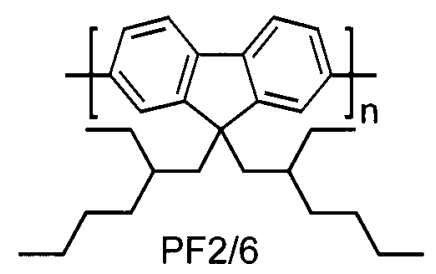

the chain backbone. PF are important materials since they have potential applications for polymer-based lightemitting diodes. ${ }^{7}$ The average conformation of the PF chain in solution was inferred from the form factor (small-angle-neutron and light scattering) and the translational diffusion, as discussed in section IIIA. In contrast to this semiflexible chain structure, PF was reported to adopt an extended helical conformation in the liquid crystall ine undiluted state. ${ }^{6}$ The intermediate scattering function and orientation relaxation function of nondilute solutions of PF in toluene, obtained from dynamic (isotropic and anisotropic) light scattering over a broad concentration range in the isotropic regime, are presented in sections III B1 and III B2. The chain length and concentration of the transport properties are outlined in sections IIIC and IIID, and the main results are summarized in the concluding section IV.

\section{Experimental Section}

Materials. The synthesis of poly(9,9-bis(2-ethylhexyl)fluorene-2,7-diyl), $\mathrm{PF} 2 / 6$, is described el sewhere. ${ }^{6}$ Its molecular structure is illustrated in Chart 1 , where $\mathrm{n}$ is the degree of polymerization. We used four fractions obtained from the same sample using preparative GPC. The weight-average mol ecular weight $M_{w}$ was obtained by static light scattering from di lute solutions in toluene using the refractive index contrast $\mathrm{dn} / \mathrm{dc}$ $=0.144 \pm 0.03 \mathrm{~cm}^{3} / \mathrm{g}$ at $\lambda=633 \mathrm{~nm}$. The values of $M_{w}$, polydispersity $M_{w} / M_{n}$, and the contour length $L_{w}\left(=I_{m} M_{w} / 388\right.$, with $\mathrm{Im}_{\mathrm{m}}=0.75 \mathrm{~nm}$ ) for the four fractions are listed in Table 1 .

Small-Angle Neutron Scattering (SANS). Measurements were performed in KWS II , a small-angle camera in FZ-J ülich, 
Table 1. Molecular Characteristics of the Polyfluorenes Used

\begin{tabular}{ccccccr}
\hline sample & $\begin{array}{c}\mathrm{M}_{\mathrm{w}} \\
(\mathrm{kg} / \mathrm{mol})\end{array}$ & $\mathrm{M}_{\mathrm{w}} / \mathrm{M}_{\mathrm{n}}$ & $\begin{array}{c}\mathrm{L}_{\mathrm{w}} \\
(\mathrm{nm})\end{array}$ & $\begin{array}{c}\mathrm{L}_{\mathrm{e}} \\
(\mathrm{nm})^{\mathrm{a}}\end{array}$ & $\mathrm{C}^{*}(\mathrm{~g} / \mathrm{mL})^{\mathrm{b}}$ & $\begin{array}{c}\mathrm{R}_{\mathrm{h}} \\
(\mathrm{nm})\end{array}$ \\
\hline F1 & 253 & 1.22 & 490 & 76 & $9.5 \times 10^{-4}$ & 19.3 \\
F2 & 146 & 1.22 & 280 & 58 & $1.3 \times 10^{-3}$ & 12.5 \\
F3 & 125 & 1.57 & 240 & 53 & $1.4 \times 10^{-3}$ & 11.4 \\
F4 & 89 & 1.63 & 171 & 44 & $1.7 \times 10^{-3}$ & 9.8
\end{tabular}

a Calculated from ${ }^{1,2} L_{e}=\left\{\left.2\right|^{2}\left[L / l-1+\exp \left(-L_{w} / l\right)\right]\right\}^{1 / 2} \cdot{ }^{b} C a l-$ culated from $c^{*}=M /\left(N_{A} L_{e}{ }^{3}\right), N_{A}$ being the Avogadro number.

using $\lambda=6.32 \AA$ with a spread $\Delta \lambda / \lambda=0.2$. The scattered neutrons were detected with a $2-\mathrm{D}{ }^{3} \mathrm{He}$ detector consisting of $64 \times 64$ channels, each of $0.8 \mathrm{~cm}$ width. The count rate was controlled in such a way that dead time effects were negligible. The experimental scattering intensity from the PF-F $4 / d$ toluene dilute sol ution after subtraction of the solvent contribution was converted to absolute scattering cross section (in $\mathrm{cm}^{-1}$ ) using a standard polyethylene sample cal ibrated against vanadium. Two detector distances $(2$ and $8 \mathrm{~m}$ ) from the sample were used to cover a broad q range.

Photon Correlation Spectroscopy (PCS). The desired dynamic structure factor and the orientation relaxation function of isotropic PF/toluene sol utions were computed from the experimental time correlation functions $\mathrm{G}(\mathrm{q}, \mathrm{t})$ of the polarized (VV) and depolarized (VH) light scattering intensities, respectively: ${ }^{8}$

$$
C(q, t)=[(G(q, t)-1) / f *]^{1 / 2}
$$

where $\mathrm{f}^{*}$ is an instrumental factor, $\mathrm{q}=4 \pi(\mathrm{n} / \lambda) \sin (\theta / 2)$ the scattering wavevector, and the time $t$ ranges from $10^{-7}$ to $10^{3}$ $\mathrm{s}$. In the presence of multiexponential decay the analysis of $\mathrm{C}(\mathrm{q}, \mathrm{t})$ proceeded via inverse Laplace transformation, leading to the distribution relaxation function $L(\ln \tau)$ :

$$
C(q, t)=\int_{-\infty}^{\infty} L(\ln \tau) \exp (-t / \tau) d \ln \tau
$$

The probable relaxation rates $\Gamma_{\mathrm{i}}$ of the contributing mechanisms were obtained from the peak positions of $L(\ln \tau)$, and the corresponding intensities were computed from $\mathrm{I}_{\mathrm{i}}(\mathrm{q})=$ $a_{i}(q) I(q)$, where I (q) is the total light scattering intensity (normalized to that of the neat toluene) and the amplitude is the integral $a_{i}=\int L(I n \tau) d(I n \tau)$ over the ith peak. ${ }^{9}$ All measurements were carried out at $20^{\circ} \mathrm{C}$.

Shear Rheometry. The linear viscoelastic material functions (storage modulus G' and loss modulus G") were measured with a Rheometric Scientific ARES-HR rheometer with a very sensitive transducer 100FRTN1. Depending on sample availability Couette ( $1 \mathrm{~mm}$ gap, $10 \mathrm{~mm}$ height) or parallel plates (25 mm diameter, $1 \mathrm{~mm}$ gap) geometries were utilized, whereas temperature control was achieved via a recirculating ethylene glycol/water mixture $\left(20^{\circ} \mathrm{C}\right)$. A homemade solvent trap system was used to reduce solvent evaporation problems. Measurements included dynamic frequency sweeps and were proceeded by dynamic strain and time sweeps in order to establish equilibrium conditions and linear viscoelastic response.

\section{Results and Discussion}

A. Chain Structure in Dilute Solution. Figure 1 shows the Kratky plot, I (q) $q^{2}$ vs $q$, for the fraction F4 obtained from the SANS measurements in dilute solution. The linear part of this function indicates that F4 chains appear to be rigid for $q$ higher than about 0.2 $\mathrm{nm}^{-1}$ and flexible at lower magnification. For randomly oriented cylindrical rods with Kuhn length $b=21$ (I being the persistence length) and rod diameter $d$, the Holzer plot of I $(q) q\left(\propto \exp \left(-q^{2} d^{2} / 2\right)\right)$ against $q^{2}$ in the inset of Figure 1 allows the estimation of $d=1.8 \pm 0.5$

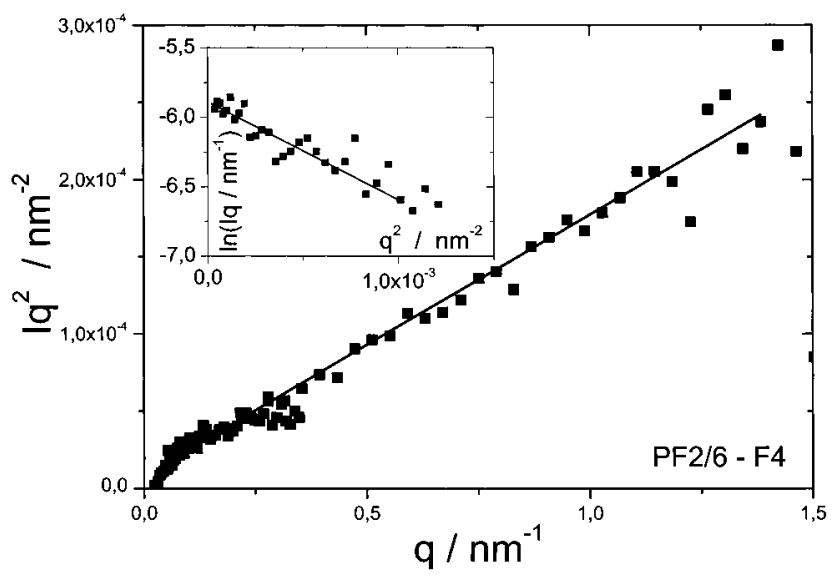

Figure 1. SANS intensity of PF $2 / 6-F 4$ fraction $(0.3$ wt \% in d-toluene). The Kratky plot indicates the distinct conformational regions of semiflexible chains whereas the Holzer plot in the inset serves for the estimation of the chain diameter.

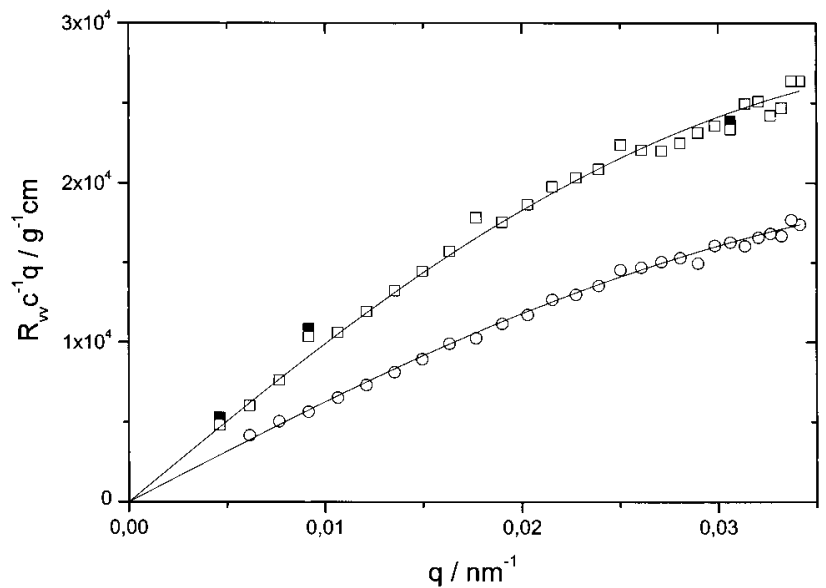

Figure 2. Distribution of the experimental reduced $R_{v v}(q) / c$ absolute Rayleigh ratio for $F 1(0.22 \mathrm{~g} / \mathrm{L}, \square)$ and $F 2(0.5 \mathrm{~g} / \mathrm{L}$, O) fractions of PF 2/6 in toluene in the Holzer representation. The solid lines denote single Gaussian chain scattering functions. The solid points for $\mathrm{F} 1$ are intensities from PCS experiments.

$\mathrm{nm}$. This value is needed for the estimation of I from the translation diffusion (see below).

The low light scattering q's are appropriate for probing the overall chain structure. F or the two highest fractions $\mathrm{F} 1$ and $\mathrm{F} 2$, the Holzer pl ot of $q R_{v v} / C$ against $q$ of Figure 2 ( $R_{v v}$ is the absolute Rayleigh ratio) conforms well to the Gaussian form factor $\mathrm{P}(\mathrm{x})=2 \mathrm{x}^{-2}\left(\mathrm{e}^{-\mathrm{x}}+\mathrm{x}-\right.$ 1) ( $x=q^{2} R_{g}{ }^{2}, R_{g}$ being the chain radius of gyration). The three experimental points (solid) of $F 1$ denote intensities obtained from PCS (eqs 1 and 2, Figure 3). The obtained values $R_{g}=32 \mathrm{~nm}$ for $F 1$ and $25 \mathrm{~nm}$ for $\mathrm{F} 2$ conform to the expression $\mathrm{R}_{\mathrm{g}}{ }^{2}(\mathrm{~L}, \mathrm{I})=(\mathrm{LI} / 3)(1-3 \mathrm{I} / \mathrm{L})$ for wormlike chains without excluded-volume effects ${ }^{1}$ using a persistence length value of $\mathrm{I}=6.5 \pm 0.5 \mathrm{~nm}$. The latter value is in accord with the Kratky plot of Figure 1.

Additional independent information on the chain conformation was extracted from the translational diffusion in dilute conditions. For wormlike chains, $\mathrm{D}_{0}=$ $(k T / 3 \pi \eta L) f^{\prime}(L / 2 I, d)$, where $f^{\prime}$ is a complicated function of $L, I$, and $d, 1,2$ determines the decay of the dynamic form factor $\mathrm{P}(\mathrm{q} \rightarrow 0, \mathrm{t})=\exp \left(-\mathrm{D}_{0} \mathrm{q}^{2} \mathrm{t}\right)^{1,2}$ The latter can be readily measured with $P C S$ in the polarized (VV) scattering geometry. Figure 3 displays unnormalized $P(q, t)$ for dilute solutions of $F 1$ and $F 4$ in toluene at $q$ 


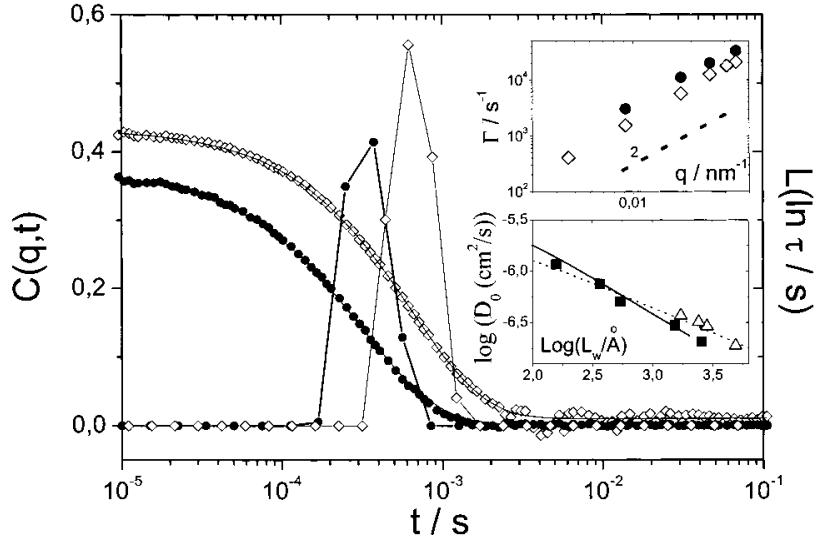

Figure 3. Dynamic self-correlation function for $\mathrm{F} 1(\diamond, 0.22$ $\mathrm{g} / \mathrm{L})$ and $\mathrm{F} 4(0,0.53 \mathrm{~g} / \mathrm{L})$ in toluene at $\mathrm{q}=9 \times 10^{-3} \mathrm{~nm}^{-1}$ represented by a single distribution relaxation function $L$ (In $\tau)$. The rate $\Gamma$ (inset) scales with $q^{2}$ indicated by the slope 2 . Solid and open symbols are respectively for F 1 and F4. The dependence of the chain translational diffusion $D_{0}$ (open triangle) on the contour length $L_{w}$ and is theoretical representation (dotted line) is shown in the lower inset. For comparison, $D_{0}$ (solid square) of poly(p-phenylene)s (ref 2) are also shown.

$=9 \times 10^{-3} \mathrm{~nm}^{-1}$ and $20^{\circ} \mathrm{C}$. Note that only about $40 \%$ of the scattering intensity arises from concentration fluctuations due to single chain diffusion at these low concentrations. The single process of $\mathrm{C}(\mathrm{q}, \mathrm{t})$ captured by the unimodal $L(\ln \tau)$ is diffusive $\left(\Gamma \propto q^{2}\right.$; see upper inset of Figure 3 ), and the extracted $D_{0}=\Gamma / q^{2}$ is depicted as a function of $L_{w}$ in the lower inset of Figure 3. As compared to the poly(paraphenylene)s, PPPs (solid squares in the lower inset of Figure 3), PF samples appear to be less rigid since for the same contour length they diffuse faster. In fact, using $\mathrm{d}=1.8 \pm 0.5 \mathrm{~nm}$ (from SANS), PF molecules can be described as wormlike chains I $=7 \pm 0.5 \mathrm{~nm}$ (dashed line in the lower inset of Figure 3), in accord with light scattering data. For a dioctyl-substituted poly(fluorene), a Kuhn segment value of $b=17 \pm 2 \mathrm{~nm}(\mathrm{l}=8 \pm 1 \mathrm{~nm})$ was reported based ${ }^{10}$ only on $\mathrm{R}_{\mathrm{g}}$ data. Also, the absorption spectra of dihexylsubstituted poly(fluorene) in solution ${ }^{11}$ led to a conjugation length of about 12 fluorene units, and hence the persistence length was at least as high as this value $(\sim 10 \mathrm{~nm})$. Finally, the difference in the chain rigidity between PPPs and PF apparently arises from the disparity in the bond angles of the adjacent monomer units $\left(0^{\circ}\right.$ and $21^{\circ}$ for PPPs and PF, respectively). Summing up, the present PF 2/6 polymers are stiff chains over a length encompassing about 18 fluorene units with a monomer length $I_{\mathrm{m}}=0.75 \mathrm{~nm}$.

B. Behavior in Nondilute Solutions. B.1. Intermediate Scattering F unction. The onset of interactions is usually marked by changes in the concentration dependence of the intensity and diffusion of the concentration fluctuations. The latter decay by cooperative chain motion, which is controlled by the enhanced osmotic modulus $(\partial \pi / \partial c)_{P, T} \propto R T c / R_{v V}$, to account for the contribution of additional processes to the static light scattering. Hence, the cooperative diffusion coefficient $\mathrm{D}_{\mathrm{c}}(\propto \partial \pi / \partial \mathrm{c})$ should increase with concentration. Figure 4 shows the variation of the normalized $D_{d} / D_{0}(a)$ and $\left(\mathrm{M}_{\mathrm{w}} / \mathrm{RT}\right)(\partial \pi / \partial \mathrm{c})_{\mathrm{P}, \mathrm{T}}(\mathrm{b})$ with the reduced concentration $\mathrm{c} / \mathrm{c}^{*}$ for the fractions F1, F2, and F4. Like in semidilute solutions of flexible homopolymers, superimposed curves are obtained for both static and dynamic properties in this representation. However, the concentration de-

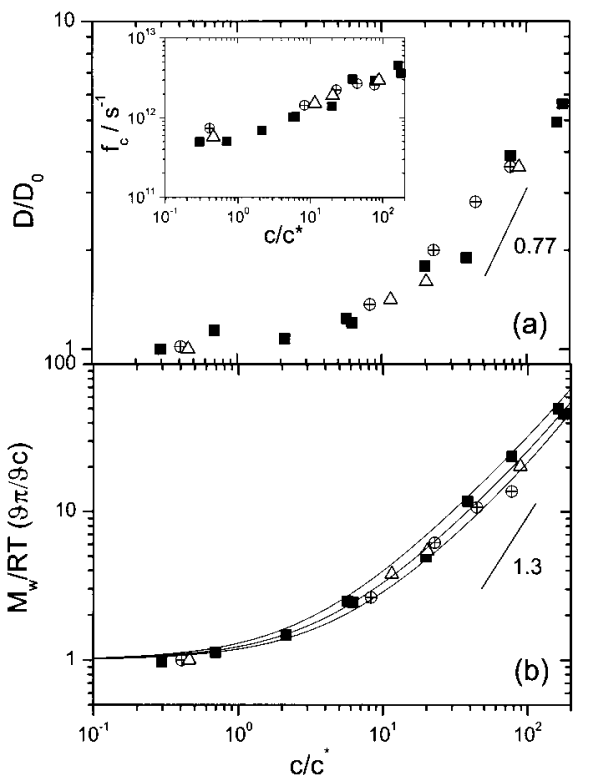

Figure 4. Normalized cooperative diffusion $D_{d} / D_{0}(a)$ and osmotic pressure $\left(\mathrm{M}_{\mathrm{w}} / \mathrm{RT}\right)(\partial \pi / \partial \mathrm{c})$ (b) vs reduced concentration c/c* for F1 (ם), F2 $(\Delta)$, and F4 $(\otimes)$ nondilute solutions in toluene. Solid lines with slopes 0.77 (a) and 1.3 (b) denote the scaling predictions for semidilute flexible homopolymer solutions. The variation of the cooperative friction coefficient $f_{c}$ with $c / C^{*}$ is shown for the three fractions in the inset. The three solid lines through the data (from left to right. F1, F2, F4) in (b) represent the fit of the scaling particle theory using $b_{\mathrm{e}} \approx$ $0.5 \mathrm{~nm}$.

pendencies are weaker than the scaling predictions (indicated in Figure 4) for flexible homopolymers. ${ }^{1,2,12}$

The normalized osmotic compressibility $\left(\mathrm{M}_{\mathrm{w}} / \mathrm{RT}\right)(\partial \pi /$ $\partial C)_{P, T}$ can be described by the scaling particle theory (SPT) ${ }^{1 \mathrm{c}}$ for wormlike spherocylinders with two virial coefficients, from which the second retains Onsager's form $\mathrm{B}=(\pi / 2)\left(\mathrm{dL}^{2} \rho+6 v\right)$ with $v=(\pi / 4)\left(\mathrm{Ld}^{2}+(2 / 3) \mathrm{d}^{3}\right)$. An adequate representation was obtained as shown by the sol id lines in Figure 4b. However, much like for the poly(p-phenylene) $s^{2 b}$ in nondilute solutions, the extracted value of the effective chain diameter $(d \approx 0.5$ $\mathrm{nm}$ ) was significantly underestimated when compared to the reported value of $d \approx 1.8 \pm 0.5 \mathrm{~nm}$ in section III.A. As a matter of fact, $d$ depends on the choice of the effective length $L$ of the fuzzy cylinder, ${ }^{1,2 d}$ representing a semistiff polymer chain under the assumption of fast local conformational transitions, compared to the overall chain motion. On the basis of the good quality of the fits and the similar low fitted values of $d$ in all three PF fractions, it is apparent that this model should use lower $\mathrm{L}$ values. This assertion is supported by the estimation of an effective $L_{e}$ from the Onsager's prediction of the concentration for the transition to the nematic state, $c_{n}=16 /\left(\pi \mathrm{dLe}_{e}^{2}\right.$ ); for $\mathrm{F} 1$ (with $\mathrm{L}_{\mathrm{e}}=76 \mathrm{~nm}$; see $L_{e}$ and $c^{*}$ values in Table 1 ) we have $c_{n}=0.37 \mathrm{~g} / \mathrm{mL}$, and hence we obtain $L_{e}=65 \pm 6 \mathrm{~nm}$, using $d \approx 1.8 \pm$ $0.5 \mathrm{~nm}$. Thus, despite the obtained good fit in Figure $4 \mathrm{~b}$, the SPT model is not self-consistent since the obtained parameters for describing the data cannot be rationalized. As this problem was also encountered with the PPPs, $2 \mathrm{~b}, \mathrm{~d}$ there seems to be a fundamental difficulty with the effective sizes of the fuzzy cylinders representing these wormlike chains that should be reconsider; one possibility is that they are concentration-dependent.

From the two main experimental quantities of Figure 4 , the extracted local cooperative friction coefficient $f_{c}$ 


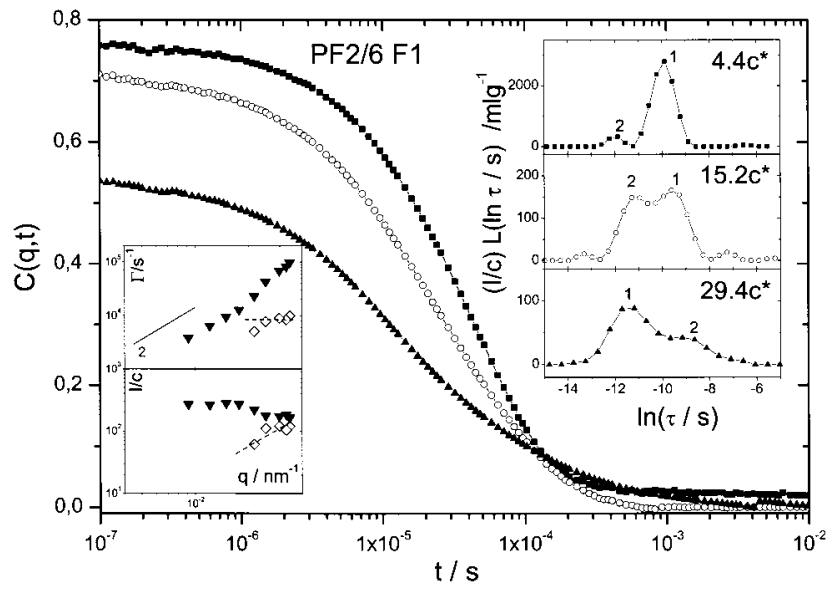

Figure 5. Intermediate scattering function $\mathrm{C}(\mathrm{q}, \mathrm{t})$ of $\mathrm{F} 1$ in toluene at $\mathrm{q}=0.034 \mathrm{~nm}^{-1}$ and three concentrations. The corresponding distribution relaxation functions multiplied by the intensity l (q)/c to facilitate the comparison among different concentrations are shown in the inset for three reduced $\mathrm{c} / \mathrm{c}^{*}$ indicated by the numbers. The lower inset displays the variation of the intensity and rate of the process (1) and (2) for the highest concentration $\left(c / c^{*}=29.4\right)$ with $q$ in a log-log presentation where the slope 2 indicates the scaling.

$=(1-\phi) \mathrm{kT}(\partial \pi / \partial \mathrm{C})_{\mathrm{P}, \mathrm{T}} / \mathrm{D}_{\mathrm{C}}$ is expectedly found to be independent of molecular weight and increasing with c. Its concentration dependence is frequently, but erroneously, identified with that of the local self-friction $f_{s}=k T / D_{s}(c)$, with $D_{s}(c)$ being the self-diffusion at finite concentrations. In fact, the rate of increase of $\mathrm{f}_{\mathrm{c}}$ (inset of Figure 4) with the normalized concentration is moderate when compared with that of $f_{s}$ in the semiflexible poly(p-phenylene) $s^{2}$, while their $f_{c}(c)$ are quite similar. ${ }^{2 d}$ The quantity $\mathrm{f}_{\mathrm{c}}$ involves both self- and crosscorrelation terms of the vel ocity of the solute particles; the latter distinguish it from $\mathrm{f}_{\mathrm{s}}$ involving only velocity self-correlation contributions. The cooperative diffusion is the dominant mechanism for the relaxation of the thermal concentration fluctuations in nondilute polymer systems. Evidence of an additional slower and weaker process in the experimental $\mathrm{C}(\mathrm{q}, \mathrm{t})$ was reported in few semiflexible polymers. 2,3 It is a diffusive mode with weak concentration dependence; its molecular origin is as yet uncertain.

The intermediate scattering function of $\mathrm{F} 1$ at $\mathrm{q}=$ $0.034 \mathrm{~nm}^{-1}$ for three concentrations is displayed in Figure 5. The corresponding distribution relaxation times (right inset of Figure 5) weighted by I(c)/c for comparison show a second peak (2) in addition to the cooperative diffusion (1) contribution. This assignment is based on the characteristics of the two processes. For example, at $c c^{*}=29.4$ the variation of the rate and intensity of (1) and (2) (left inset of Figure 5) with q identifies the former with the cooperative diffusion which is further documented by its concentration dependence (Figure 4). As to process (2), it displays a correlation hole at $\mathrm{q} \rightarrow 0$ since it becomes more important with increasing $q$, whereas its characteristic rate has a purely relaxational nature, i.e., $\Gamma_{2} \propto q^{0}$. At low concentrations $\Gamma_{2}$ exceeds the rate $\Gamma_{c}=D_{c} q^{2}$ (for the relaxation of the concentration fluctuations via cooperative diffusion of the network), and it becomes slower at high concentrations (right inset of Figure 5). These distinct features of the second contribution to $\mathrm{C}(\mathrm{q}, \mathrm{t})$ are, to our knowledge, for the first time reported for nondilute semiflexible synthetic polymers. ${ }^{5} \mathrm{~A}$ second process that becomes stronger and slower with solute concen- tration was reported for solutions of the rigid ( $\alpha$-helix) poly ( $\gamma$-benzyl- $\alpha$-L-glutamate). ${ }^{3 c}$ This process was faster than the cooperative diffusion rate and became more important with increasing q. Its rate, however, depended on $\mathrm{q}$ in contrast to the present case and was assigned to the contribution of both rotational and translational motion (see eq 3).

For dilute solutions ${ }^{13}$ of flexible and rigid polymers,

$C(q, t)=S_{0}\left(x^{\prime}\right) \exp \left(-D q^{2} t\right)+S_{2}\left(x^{\prime}\right) \exp \left[-\left(\Gamma_{2}+D q^{2}\right) t\right]$

where the intensity $S_{0}\left(x^{\prime}\right)$ and $S_{2}\left(x^{\prime}\right)$ decreases and increases, respectively, with $x^{\prime} \equiv \mathrm{qR}$. $\Gamma_{2}$ depends on the chain rigidity and may be identified with either rod rotational diffusion or the inverse longest time $\tau_{1}$ for conformational relaxation of a flexible chain. Only for $\Gamma_{2} \gg \mathrm{Dq}^{2}$ (over the considered $\mathrm{q}$ range), the decay rate of the second exponential becomes q-independent and is al ways faster than the diffusive decay rate $D q^{2}$ of the first exponential in eq 3; this prediction is valid at low concentrations in the early semidilute regime (see also the upper left inset of Figure 5). For interacting nondilute semiflexible polymers, there is no rigorous theory for $\mathrm{C}(\mathrm{q}, \mathrm{t})$ to quantitatively account for the new data of Figure 5 .

In solutions of entangled polymers viscoelastic relaxation can be an alternative contribution 4,5 to $C(q, t)$. Polymer concentration fluctuations can induce stress into the entangled network (coupled via volume interactions ${ }^{4 b}$ ), and hence compl ete relaxation of the former can proceed via chain conformational relaxation with characteristic time $\tau_{1}$. Cooperative diffusion is always the faster process, whereas the slower q-independent rate is $\Gamma_{2} \approx 1 / \tau_{1}$. Theoretical consideration of the correlation function $\mathrm{C}(\mathrm{q}, \mathrm{t})$ of concentration fluctuations in polymer solutions in the entangled regime assuming validity of the reptation model (motion along a tube) leads to a three-step relaxation function $4 \mathrm{~b}$

$$
C(q, t)=A_{c} C_{c}(q, t)+A_{R} C_{R}(q, t)+A_{r e p} C_{r e p}(q, t)
$$

for $q$ larger than a (low) threshold $q_{m}\left(q_{m}{ }^{2} \tau_{1} \approx 1\right)$, where the three amplitudes and decay functions stand for cooperative diffusion, Rouse (tube length fluctuations), and reptation (tube conformation). The variation of the rate $\Gamma_{\text {rep }}$ and the amplitude $A_{\text {rep }}$ with q depends on the magnification $x^{\prime}=\mathrm{qR}$; for $\mathrm{x}^{\prime}<1, \Gamma_{\text {rep }} \sim 1 / \tau_{1}$ and $A_{\text {rep }} \propto q^{0}$, whereas for $L>q^{-1}>R, A_{\text {rep }}$ increases with q. $A_{R}$ is low compared to $A_{\text {rep }}$ in the available q range, and hence this contribution in eq 4 can be neglected.

To contrast the theoretical and experimental behavior of the second process of $C(q, t)$ we need an estimate of $\tau_{1}$ and the entanglement molecular mass $\mathrm{M}_{\mathrm{e}}$ of PF for an assessment of the extent of the entanglements in the studied solutions. Figure 6 shows the storage ( $\left.G^{\prime}\right)$ and loss ( $\left.G^{\prime \prime}\right)$ moduli as a function of frequency $\omega$ for solutions of $\mathrm{F} 1$ at two different concentrations, 15\% and 23.2 wt \% in toluene. On the basis of these linear viscoelastic data, both solutions formed a transient physical network ( $\left.G^{\prime}>G^{\prime \prime}\right)$ at high frequencies, while displaying liquidlike behavior ( $\left.G^{\prime}<G^{\prime \prime}\right)$ at low frequencies. For the $15 \% \mathrm{~F} 1$ soluti on the standard terminal flow scaling behavior $\left(\mathrm{G}^{\prime} \sim \omega^{2}, \mathrm{G}^{\prime \prime} \sim \omega\right)$ was reached. The extrapolation of these terminal $G^{\prime}$ and $G^{\prime \prime}$ lines until they intersect defines the longest chain relaxation time $\tau_{1}=1 / \omega_{\mathrm{c}}$ with $\omega_{\mathrm{c}}$ being the crossover frequency. However, this intersection occurs at slightly different fre 


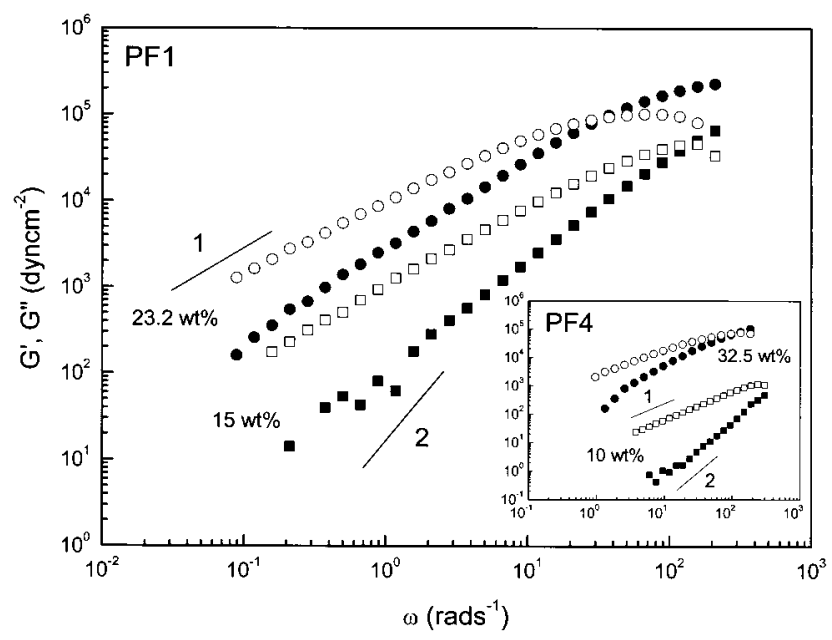

Figure 6. F requency dependence of the dynamic shear moduli (storage G' $\square, 0$; loss $G^{\prime \prime} \mathbf{\square}, 0$ ) for two $F 1$ solutions in toluene (15 wt \%, squares; 23.2 wt \%, circles) at $25^{\circ} \mathrm{C}$. The slopes indicate the scaling of the terminal regime. The $\mathrm{G}^{\prime}, \mathrm{G}^{\prime \prime}$ data for two $\mathrm{F} 4$ solutions are shown in the inset (10 wt \%, squares; 32.5 wt $\%$, circles).

quency than the crossover frequency apparently due to the finite polydispersity; for consistency, we used the extrapolated values for all data sets. For the more concentrated $\mathrm{F} 1$ solution, the terminal flow was reached at the lowest frequencies region and expectedly the crossover frequency was significantly slower than in the $15 \% \mathrm{~F} 1$ solution. The dynamic mechanical behavior of the lowest fraction $\mathrm{F} 4$ is displayed in the inset of $\mathrm{F}$ igure 6 for two concentrations in toluene. From the information obtained in the frequency range covered, the $10 \mathrm{wt}$ $\% \mathrm{~F} 4$ solution appears to be unentangled. The entanglement molecular mass was estimated from $\mathrm{G}^{\prime}, \mathrm{G}^{\prime \prime}$ data of the melt $\mathrm{F} 3$ to amount to $\mathrm{M}_{\mathrm{e}}=12 \pm 4 \mathrm{~kg} / \mathrm{mol}$ (upper limit). On the basis of this value, the $F 1$ and $F 4$ solutions become entangled above about $6 \%$ and $14 \%$ in accordance with the data of Figure 6.

In the framework of eq 4, the second viscoelastic relaxation process should be observed in well entangled polymer solutions with $\Gamma_{2}$ being slower than $\Gamma_{c}=D_{c} q^{2}$, the rate of the relaxation of the concentration fluctuations driven by the osmotic pressure of the polymer network. F or the PF fractions F 1, F2, and F 4, the second contribution is resolved for concentrations lower than the entanglement concentration $\mathrm{C}_{\mathrm{e}} \approx \mathrm{Me}_{\mathrm{e}} / \mathrm{M}$ and moreover $\Gamma_{2}>\Gamma_{\mathrm{c}}$ as shown for $\mathrm{F} 1 \mathrm{1.44 \%}\left(\mathrm{c} / \mathrm{C}_{\mathrm{e}} \approx 0.2\right)$ in Figure 7; the slow decay at $\mathrm{t}>0.01 \mathrm{~s}$ is attributed to cluster scattering, ${ }^{2 \mathrm{e}}$ which is no further considered in this work. The correlation $\mathrm{C}(\mathrm{q}, \mathrm{t})$, its distribution relaxation function $\mathrm{L}(\ln \tau)$ at two wavevectors $\left(\mathrm{q}_{\mathrm{L}}=0.034 \mathrm{~nm}^{-1}\right.$ and $\mathrm{q}_{\mathrm{s}}$ $=0.012 \mathrm{~nm}^{-1}$ ), and the rates $\Gamma_{c_{1}} \Gamma_{2}$ (left inset of Figure 7) demonstrate the two-step decay and the distinctly different $q$ dependence of the two rates. Unlike the situation at high concentrations (Figure 5, left inset), $\Gamma_{2}>\Gamma_{\mathrm{c}}$ at this lower concentration. The reduced intensity $\mathrm{I}_{2} / \mathrm{C}$ of the second process in three PF samples varies with $\mathrm{qL}$ as shown in the right inset of Figure 7. F or the lowest fraction $\mathrm{F} 4$ (and hence low $\mathrm{qL}$ ) $\mathrm{I}_{2}$ becomes insensitive to $\mathrm{qL}$ variations. This behavior in the unentangled regime can qualitatively be captured by eq 4 of the entangled regime, but not eq 3 .

Alternatively, an assessment of the experimental data (especially for the lower concentrations where the second process is detected, e.g., 1.44 wt \% of $\mathrm{F} 1$ in Figure 7) in the framework of the Doi-Onuki approach which

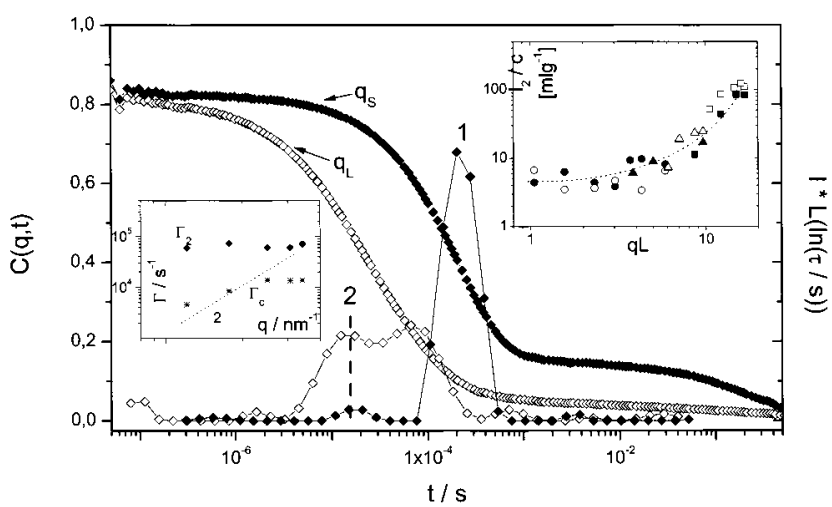

Figure 7. Intermediate scattering function $\mathrm{C}(\mathrm{q}, \mathrm{t})$ of $1.44 \%$ $\mathrm{F} 1$ solution in toluene at large $\mathrm{q}_{\mathrm{L}}=0.034 \mathrm{~nm}^{-1}$ and low $\mathrm{q}_{\mathrm{s}}=$ $0.012 \mathrm{~nm}^{-1}$ along with the corresponding distribution relaxation functions $I L(\ln \tau)$. The $q$ dependence of the two rates $\Gamma_{C}$ and $\Gamma_{2}$ for this concentration and the intensity $\mathrm{I}_{2} / \mathrm{C}$ for the second process for the three fractions F1, F2, and F4 at different concentrations are given in the insets (left and right, respectively).

accounts for the coupling of stress and concentration fluctuations without explicit requirement for a reptation mechanism, but with implicit consideration of entangled systems forming a transient rubbery plateau, ${ }^{4 c, e}$ was not successful; in particular, for the F 1 samples at the two higher concentrations of Figure 5, the q-dependencies of the experimental relaxation rates $\Gamma_{c}$ and $\Gamma_{2}$ were not recovered, whereas the ratio of osmotic to shear modulus was unrealistic in some cases; this ratio was determined by the amplitudes of the cooperative and the viscoelastic modes in $\mathrm{C}(\mathrm{q}, \mathrm{t})$.

It is thus clear that a more systematic assessment of these theories for the viscoelastic mode, particularly for semiflexible polymers, requires a much wider range of molecular weights and concentrations than available in this work (the latter because of the interference of the orientation fluctuations at high concentrations, as discussed below) and is beyond the scope of the current investigation.

Therefore, the rigorous theory for $\mathrm{C}(\mathrm{q}, \mathrm{t})$ of interacting nondilute and entangled flexible polymers predicts and fully characterizes the second viscoelastic contribution, but the comparison with the experience (Figures 5 and 7) is unsatisfactory. It seems that the solutions studied were not concentrated enough, pointing for a need for theoretical support for the early semidilute regime. In addition, a consideration a the stress-diffusion coupling could be insufficient, possibly missing some other mechanisms in the semiflexible systems. Nevertheless, from the experimental evidence it is cleat that the second process can be attributed to the chain conformational relaxation mainly because of the molecular mass de pendence of $\Gamma_{2}$ and its comparison with the shear relaxation rate (Figures 6 and 9). Further, $\Gamma_{2}$ expectedly decreases with $c$ in contrast to the cooperative diffusion $D_{c}$ (Figures $4 a$ and 5), and hence the separation of the rates $\Gamma_{c}$ and $\Gamma_{2}$ increases with $c$. However, the desired measurements of $\mathrm{C}(\mathrm{q}, \mathrm{t})$ at even higher concentrations are affected by the contribution of the anisotropic scattering, which will be discussed next followed by the concentration dependence of $\Gamma_{2}$.

B.2. Orientation Relaxation. As already mentioned, polyfluorenes (Chart 1) possess significant inherent optical anisotropy only along the chain backbone. Dynamic depolarized light scattering was detectable above $2.8 \%$ for $\mathrm{F} 1$ only at high q's. At this concentration 


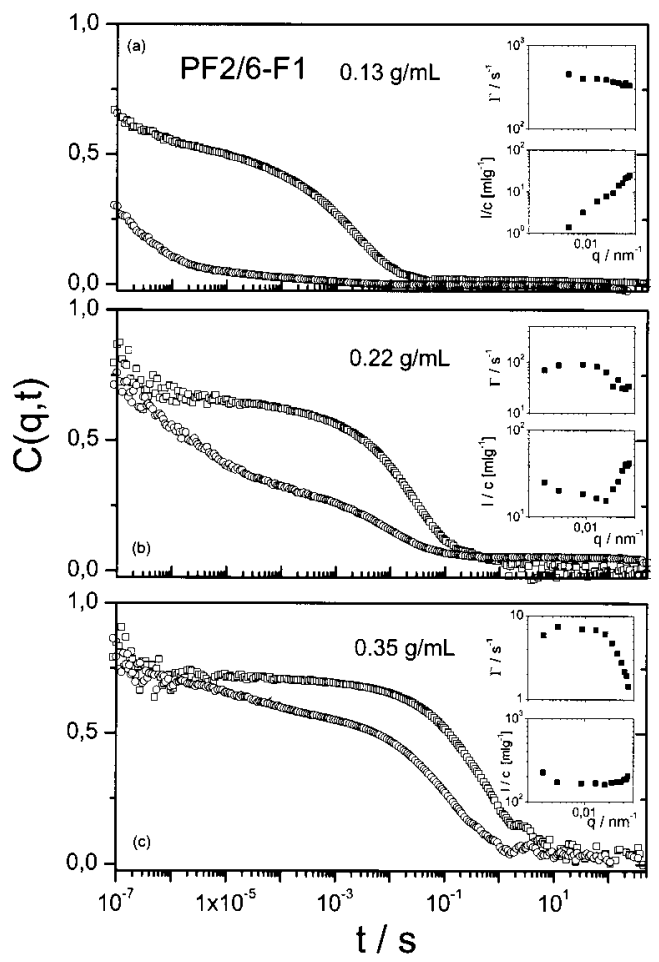

Figure 8. Experimental orientation relaxation function of $F 1$ solutions in toluene at three concentrations, (a) 0.13 , (b) 0.22 , and (c) $0.35 \mathrm{~g} / \mathrm{mL}$ and at $\mathrm{q}=0.034$ ( $\square$ ) and $4 \times 10^{-3} \mathrm{~nm}^{-1}(O)$. The variation of the intensity and the rate of the slow process vs $q$ at each concentration is shown in the insets (lower and upper, respectively).

$\left(c / c^{*}=17.5\right)$, the orientation relaxation function $C_{o r}(q, t)$ at $\mathrm{q}=0.034 \mathrm{~nm}^{-1}$ was nonexponential, conforming to

$$
\mathrm{C}_{\text {or }}(\mathrm{q}, \mathrm{t})=\alpha(\mathrm{q}) \exp \left[-(\mathrm{t} / \tau)^{\beta}\right]
$$

where the shape parameter $\beta=0.54 \pm 0.05$ and the rate $\Gamma(=1 / \tau)=1.4 \times 10^{4} \mathrm{~s}^{-1}$ as compared to $\Gamma_{2}=9 \times$ $10^{3} \mathrm{~s}^{-1}$ at the same concentration. The amplitude $\alpha(\mathrm{q})$ decreased with decreasing $\mathrm{q}$, prohibiting $\mathrm{VH}$ measurements at q's below $0.025 \mathrm{~nm}^{-1}$. To use the available $\mathrm{q}$ range, higher concentrations were considered.

Figure 8 provides a compilation of experimental $\mathrm{C}_{\text {or }}(\mathrm{q}, \mathrm{t})$ at three concentrations and two q's $\left(4 \times 10^{-3}\right.$ and $0.034 \mathrm{~nm}^{-1}$ ) for each. The bimodal q-dependent shape of $C_{o r}(q, t)$ with the peculiar q dependence of the rate $\Gamma_{\mathrm{s}}$ and intensity $I_{\mathrm{s}}$ for the slow nonexponential process are the strong experimental findings. $\Gamma_{\mathrm{s}}$ decreased with q at all examined concentrations, while $I_{s}$ increased monotonically with q up to about $15 \%$, and the bimodal shape of $\mathrm{C}_{\text {or }}(\mathrm{q}, \mathrm{t})$ persisted up to the transition to nematic state $\left(c_{\mathrm{n}} \simeq 0.37 \mathrm{~g} / \mathrm{mL}\right)$. This q dependence of $\mathrm{I}_{\mathrm{s}}$ implies a crossover behavior between 0.13 and $0.22 \mathrm{~g} / \mathrm{mL}$, well bel ow $\mathrm{c}_{\mathrm{n}}$. The reduced $\mathrm{I}_{\mathrm{s}} / \mathrm{c}$ (insets) increased significantly with c only at the last concentration in the vicinity of $c_{n}$, accompanied by the strongest decrease of $\Gamma_{s}$ with q. As expected, the rate $\Gamma_{s}$ decreased strongly with $\mathrm{c}$ (insets of Figure 8), whereas the shape parameter $\beta=0.45 \pm 0.05$ was virtually c-independent. The value of $\Gamma_{\mathrm{s}}$ at $\mathrm{q} \rightarrow 0$ (obtained by linear extrapolation) compared well with the overall rotation rate $\tau_{1}^{-1}$ of the chain from rheological data (see Figure 9).

The fast process gained intensity at low q's and compensated roughly the cocurrent decrease of $I_{s}$ since the total depolarized scattering intensity was virtually q-independent. The fast dynamics falled at the edge of the PCS time window, and only at the two highest concentrations $(0.26$ and $0.35 \mathrm{~g} / \mathrm{mL})$ it became slow enough to be resolved reliably. At these concentrations, the fast decay was nonexponential and could be represented by eq 5 using $\beta_{\mathrm{f}} \approx 0.4$ for the $\mathrm{C}_{\mathrm{or}}(\mathrm{q}, \mathrm{t})$ at the low $\mathrm{q}$ of Figure $8 \mathrm{c}$. At q $=4 \times 10^{-3} \mathrm{~nm}^{-1}$ and $\mathrm{c}=0.35 \mathrm{~g} / \mathrm{mL}$ the most probable times (at the peak maxima of $\mathrm{L}$ ( $\mathrm{In}$ $\tau)$ ) were $\tau_{\mathrm{f}}=4 \times 10^{-5} \mathrm{~s}$ and $\tau_{\mathrm{s}}=0.14 \mathrm{~s}$.

A bimodal $C_{\text {or }}(q, t)$ was first observed for poly(pphenylene) (PPP) with larger persistence length $(I \approx 20$ $\mathrm{nm})$, bearing also bulky phenyl side groups. ${ }^{2 a}$ The main slow process was broader $\left(\beta_{\mathrm{s}} \approx 0.35 \pm 0.05\right)$, exhibiting similar peculiar q dependence for $\Gamma_{\mathrm{s}}$. Since $\Gamma_{\mathrm{s}}$ was much faster than the overall rotational rate $\tau_{1}{ }^{-1}$, the slow process in the $C_{\text {or }}(q, t)$ of PPP was attributed to collective Kuhn segment reorientation. The fast process of $\mathrm{C}_{\text {or }}(\mathrm{q}, \mathrm{t})$ was characterized by a narrower distribution $\left(\beta_{\mathrm{f}} \approx 0.65\right)$ and was about 2 orders of magnitude faster. The present PF polymers are less rigid $(I \approx 7 \mathrm{~nm})$, have optically isotropic alkyl side groups, possess comparably lower molar mass polydispersity and eventually form a lyotropic phase at high enough concntrations. The bimodality of $C_{\text {or }}(q, t)$ appears to be less sensitive to chain rigidity and polydispersity differences between PPPS and PF, as suggested by the situation in a PPP mixture. ${ }^{2 a}$ The different relation between $\Gamma_{\mathrm{S}}$ and $\tau_{1}{ }^{-1}$ in the two systems, as well the presence of Iyotropic phase in PF, but not PPP's, may have their origin in packing effects due to the planar monomer unit structure of the former.

In the next section we examine the dependence of the second slow processes of the intermediate scattering function (section III.B.1) and $\mathrm{C}_{\text {or }}(\mathrm{q}, \mathrm{t})$ on the chain length. This information will provide further insight into the nature of these processes.

C. Chain Length Dependence. The cooperative diffusion process $C(q, t)$ was observed for all PF fractions, and its characteristics (intensity and diffusion $D_{c}$ ) were virtually insensitive to chain length variations in the pl ot of Figure 4. The contribution of the second process to the intermediate scattering function $C(q, t)$ (section $B .1$ ) became stronger with increasing $L$ as shown in the right inset of Figure 7 , while its relaxation rate $\Gamma_{2}$ decreased with $L$ (right inset of Figure 9 below). F or all fractions and concentrations above about $0.1 \mathrm{~g} / \mathrm{mL}, \mathrm{I}_{2}$ falled within the range of the anisotropic contribution $\mathrm{I}_{\mathrm{VH}}$ to the polarized light scattering, i.e., $\mathrm{IVV}_{\mathrm{VV}}=\mathrm{I}_{\mathrm{ISO}}+$ $4 / 3 \mathrm{IHH}_{\mathrm{V}}$.

The total depolarized intensity $\mathrm{I}_{\mathrm{VH}} \propto \mathrm{N}\left\langle\gamma^{2}\right\rangle \propto \mathrm{C}\left\langle\gamma^{2}\right\rangle / \mathrm{L}$, where $\mathrm{N}$ is the number of chains and $\left\langle\gamma^{2}\right\rangle$ the average (over all chain conformations) optical anisotropy of the chain; the proportionality to $c$ (bel ow $c_{n}$ ) is valid in the absense of orientational correlations. Note that $\left\langle\gamma^{2}\right\rangle / \mathrm{L}$ $\propto L^{a}$, where $a=0$ for flexible Gaussian chains and $a=$ 1 for rigid rods. ${ }^{2 f, 8}$ At $c=6 \%, I_{\mathrm{VH}} / \mathrm{c}$ assumed within experimental error the same value for all four PF fractions, and the effective optical anisotropy per monomer unit $\left\langle\gamma^{2}\right\rangle / \mathrm{P}$ ( $\mathrm{P}$ is the number of monomers per chain) was about 600 times larger than the optical anisotropy of toluene. The constant $\left\langle\gamma^{2}\right\rangle / \mathrm{P}$ for the four fractions conforms to a rather low value of the persistence length. ${ }^{2 f}$ Furthermore, assuming 17 fluorene units per Kuhn segment (section III.A) the optical anisotropy of the fluorene unit is about $600 / 17^{2} \approx 2$ times the optical anisotropy of the toluene. Given the planar structure of the monomer unit (Chart 1 ), this is a reasonable value. 


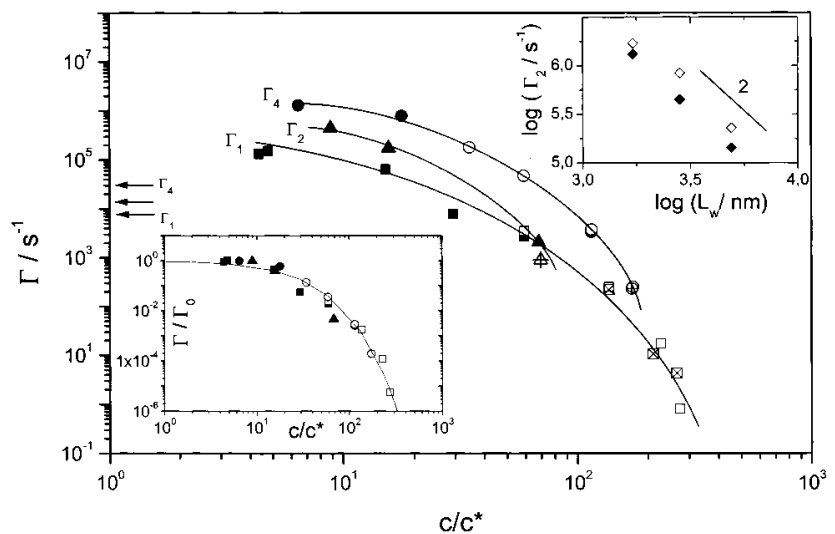

Figure 9. Chain orientation relaxation rate $\Gamma_{2}$ (solid symbols), $\Gamma_{\mathrm{s}}$ (open symbols) and $\tau_{1}{ }^{-1}$ from shear rheology (crossed symbols) of F1 (squares), F2 (triangles), and F 4 (circles) polyfluor ene fractions as a function of the reduced concentration. The horizontal arrows at low $c / c^{*}$ values denote the predictions of eq 6 for $\Gamma \approx 6 D_{\text {r }}$ of the three fractions $F 1, F 2$, and $\mathrm{F} 4$ from bottom to top, respectively. Lower inset: normalized orientation times $\Gamma / \Gamma_{0}$ vs reduced concentration $c / c^{*}$, along with their representation (solid curve) by eq 7. Upper inset: orientation rate $\Gamma_{0}$ (open symbols) at the lowest concentration for the three PF fractions vs contour length $L_{w}$. The solid symbols denote the computed values of $\Gamma_{0}$ based on the hydrodynamic radius for the three samples; the slope of -2 represents the approximate scaling for flexible chains in good solvent.

The orientation relaxation function $\mathrm{C}_{\text {or }}(\mathrm{q}, \mathrm{t})$ (section III.B.2) was also measured for $\mathrm{F} 2$ and $\mathrm{F} 4$ solutions at different concentrations. $\mathrm{C}_{\text {or }}(\mathrm{q}, \mathrm{t})$ displayed qual itatively the same features as for F 1 (Figure 8). Quantitatively, the main slow process was clearly faster in $\mathrm{F} 2$ and even faster in F4 when compared at the same concentration with $F 1$, whereas the fast contribution to the $\mathrm{C}_{\text {or }}(\mathrm{q}, \mathrm{t})$ appeared to depend much less on the chain length, falling into the short time edge of the photon correlation spectroscopy; the transition to the nematic state occurred at about $0.3 \mathrm{~g} / \mathrm{mL}$ for $\mathrm{F} 4$. At $0.29 \mathrm{~g} / \mathrm{mL}$ (still in the isotropic state but close to the transition) the fast process was exhibiting a significant slowing down. At this concentration and at $\mathrm{q}=9 \times 10^{-3} \mathrm{~nm}^{-1}$, the fast time was $\tau_{\mathrm{f}} \approx 4.6 \times 10^{-6} \mathrm{~s}$ and the slow $\tau_{\mathrm{s}} \approx 3 \times 10^{-4} \mathrm{~s}$, yiel ding a ratio $\tau_{\mathrm{s}} / \tau_{\mathrm{f}}$ which was smaller than in $\mathrm{F} 1$ near the transition. This sudden slowing down of the fast process in $\mathrm{C}_{\text {or }}(\mathrm{q}, \mathrm{t})$ reflects pretransitional effects. Moreover, the fact that $\mathrm{C}_{\text {or }}(\mathrm{q}, \mathrm{t})$ did not reach the value of 1 at short times, despite the increase of $\mathrm{I}_{\mathrm{VH}}$, corroborates the notion that the fast process is rather induced in the vicinity of $\mathrm{C}_{\mathrm{n}}$.

D. Concentration Dependence. The variation of the chain orientation time as obtained from isotropic $\left(\Gamma_{2}\right)$ and anisotropic $\left(\Gamma_{\mathrm{s}}\right)$ scattering with PF 2/6 concentration in toluene at $20^{\circ} \mathrm{C}$ is visualized in Figure 9 for three different fractions. Note the good agreement with the rheological data. At the lowest concentration, $\Gamma_{2}$ is about an order of magnitude faster in $\mathrm{F} 4$ than in $\mathrm{F} 1$, and its dependence on $L_{w}$ is depicted in the right inset of Figure 9. For wormlike chains ${ }^{1,2 d}$

$$
\mathrm{D}_{\mathrm{r}}=3 \mathrm{k}_{\mathrm{B}} \mathrm{T} /\left(\pi \eta_{\mathrm{s}} \mathrm{L}^{3}\right) \mathrm{F}(\mathrm{L} / 2 \mathrm{l}, \mathrm{d} / \mathrm{l}, \mathrm{I} / \mathrm{m} / \mathrm{l})
$$

where $\mathrm{F}$ is a function of the ratios of the contour length, diameter, and monomer length to the persistence length. The predicted ratio of the $\Gamma_{2}$ values of the two PF fractions is about 5 as indicated by the arrows in Figure 9 denoting the computed rotational rates $\left(\Gamma \approx 6 D_{r}\right)$ from eq 6. The strong $L_{w}$ dependence of $\Gamma_{2}$, within experimental uncertainty, is better captured by the overall rotational rate $\left(\mathrm{k}_{\mathrm{B}} \mathrm{T} / \eta_{\mathrm{s}}\right) /\left(4 \pi \mathrm{R}_{\mathrm{h}}{ }^{3} / 3\right)$, using the experimental hydrodynamic radii of Table 1 , as shown in the inset of Figure 9.

The orientation rates of Figure 9 can be superimposed on a single curve when plotted as normalized rate (to their corresponding values at the lowest concentration) against the reduced concentration $\mathrm{c} / \mathrm{c}^{*}$ (left inset of Figure 9). Both rigid-rod and flexible polymers would conform to such superpositi on based on scaling models. However, the tube model for rigid-rod rotation would predict a $\mathrm{c}^{-2}$ dependence above $\mathrm{c}^{*}$, whereas the reptation model for flexible entangled polymers $D_{r} \sim c^{-1.8}$. The experimental rates of Figure 9 indicate a clearly steeper dependence on concentration. In the "fuzzy" cylinder model of wormlike polymers the normalized rotational diffusion reads

$$
\frac{D_{r}}{D_{r, 0}}=\left(1+\beta_{r}^{-1 / 2} L_{e}^{4} \rho f_{r}\left(b_{e} / L_{e}\right) \sqrt{\frac{D_{r, 0}}{6 D_{\|}}}\right)^{-2}
$$

where $D_{\|}$is the axial translational diffusion, $f_{r}\left(b_{e} / L_{e}\right)$ is a function of the fuzzy cylinder aspect ratio, and the constant $\beta_{\mathrm{r}}$ reflects the maximum crowding of the chains in a cube of size $L_{e}$ that the system tolerates before loosing mobility; $b_{e}=\left(\left\langle\mathrm{H}^{2}\right\rangle+b^{2}\right)^{1 / 2}$ is the effective diameter of the fuzzy cylinder with the mean square distance between the chain midpoint and the end-toend axis. ${ }^{1}$ Theor etically, $\beta_{\mathrm{r}}=1350$ for infinitely thin rods, whereas flexibility is expected to increase this value. Further, to fit eq 7 to the experimental data of Figure 9, a concentration dependence of the parallel diffusion coefficient $D_{\|}(c)$ should be assumed. This was done in the framework of the "hole" model, where longitudinal motion of the chain occurs only when a "hole" of a volume larger than $V_{\text {ex }}{ }^{*}$ exists around it. $V_{e x}{ }^{*}$ $=\mathrm{f}\left(\lambda * \mathrm{~L}_{e}, \lambda * \mathrm{~b}_{\mathrm{e}}, \mathrm{L}, \mathrm{d}\right)$ represents the excluded volume of the chain, and $\lambda^{*} \ll 1$ expresses the effectiveness of the head-to-head collisions in slowing down $D_{\|}$with increasing concentration. The fit of eq 7 describes the experimental concentration dependence of $\Gamma / \Gamma_{0}$ (left inset of Figure 9) with adjustable parameters $\beta_{\mathrm{r}}=13500 \pm 70$ and $\lambda^{*}=0.037 \pm 0.002$. As expected, $\beta_{\mathrm{r}}$ is higher than in the rod limit and $\lambda^{*}$ is within error the same in $\mathrm{F} 1$ and F 4. Moreover, the comparison with the corresponding values $\left(\beta_{\mathrm{r}}=2000\right.$ and $\left.\lambda^{*}=0.1\right)$ in PPPs ${ }^{2 \mathrm{~d}}$ (with a smaller range of $L_{w}$ compared to the present PFs) reflects the higher flexibility of the present PF macromolecules, as also confirmed by the persistence lengths.

\section{Concluding Remarks}

The investigation of the interplay of chain conformation and dynamics in shape persistent macromolecules, and in particular the present newly synthesized polyfluorenes, was carried out using a combination of dynamic light scattering and small-angle neutron scattering experiments, complemented by shear rheometry measurements. The rotational and translational diffusion dynamics were studied over a broad concentration range up to the lyotropic state. On the basis of dilute solution measurements, the single chains were de scribed as wormlike with a low persistent length I = 7 $\pm 0.5 \mathrm{~nm}$ and cross-section diameter $\mathrm{d} \simeq 2 \mathrm{~nm}$. In the semidilute concentration regime, the rate of increase of the cooperative diffusion and the osmotic pressure with $c / c^{*}$ ( $c^{*}$ being the overlapping concentration) was 
lower compared to linear flexible chains in good solvent. The intermediate scattering function displayed a second process which was identified with the chain relaxation, based on its characteristic features and additional support from rheological data. The observed bimodal shape of the orientation correlation function was in accord with earlier findings in similar wormlike polymers, and as such it served as an identifying feature of shape persistent polymers. Finally, the fuzzy cylinder model adequately represented the concentration dependence of the osmotic modulus and the chain orientation time with fitting conformational parameters which corroborated the wormlike character of these polymers.

Acknowledgment. We are grateful to Prof. Dr. K. Müllen for his generous support and fruitful discussions, to Ms. A. Larsen for her assistance with the measurements, and to Dr. G. Petekidis for his help with the fitting analysis. The financial support of the EU (Grant HPRN-CT-2000-00003), Sony International (Europe) GmbH, Advanced Technology Center (Stuttgart), and the Greek Minstry of Education (Grant 1090-Applied Molecular Spectroscopy) is gratefully acknowledged.

\section{References and Notes}

(1) Tracy, M. A.; Pecora, R. Annu. Phys. Chem. 1992, 43, 525. Russo, P. S. In Dynamic Light Scattering; Brown, W., Ed.; Clarendon Press: Oxford, U.K., 1993. Sato, T.; Teramoto, A. Adv. Polym. Sci. 1996, 24, 6220.

(2) (a) Petekidis, G.; Vlassopoulos, D., Fytas, G.; Rülkens, R.; Wegner, G. Macromolecular 1998, 31, 6129. (b) Petekidis, G.; Vlassopoulos, D.; Fytas, G.; Rülkens, R.; Wegner, G.; Fleischer, G. Macromolecules 1998, 31, 6139. (c) Petekidis, G. Fytas, G.; Scherf, U.; Müllen, K.; Fleischer, G. J . Polym. Sci., Polym. Phys. Ed. 1999, 37, 2211. (d) Petekidis, G. Vlasso- poulos, D.; Fytas, G.; Fleischer, G.; Wegner, G. Macromolecules 2000, 33, 9630. (e) Petekidis, G.; Vlassopoulos, D.; Fytas, G.; Kountourakis, N.; Kumar, S. Macromol ecules 1997, 30, 919. (f) Petekidis, G.; Vlassopoulos, D.; Galda, P.; Rehahn, M.; Ballauff, M. Macromol ecules 1996, 29, 6832.

(3) De Long, L. N.; Russo, P. S. Macromolecules 1991, 24, 6139. Ohshima, A.; Yamagato, A.; Sato, T.; Teramoto, A. Macromolecules 1999, 32, 8645. Phalankornkul, J . K.; Gast, A. P.; Pecora, R. Macromolecules 1999, 32, 312.

(4) 4. (a) Brochard, F. J . Phys. (Paris) 1983, 44, 39. (b) Semenov, A. N. Physica A 1990, 166, 263. (c) Doi, M.; Onuki, A. J . Phys. II 1992, 2, 1631. (d) Milner, S. T. Phys. Rev. E 1993, 48, 3674. (e) Einaga, Y.; Karube, D. Polymer 1998, 40, 157.

(5) 5. (a) Adam, M.; Delsanti, M. Macromol ecules 1985, 18, 1770 (b) Nemoto, N.; Koike, A.; Osaki, K. Macromolecules 1996, 29, 1445. (c) Wang, C. H.J . Chem. Phys. 1995, 102, 6537. (d) J ian, T.; Vlassopoulos, D.; Fytas, G.; Pakula, T.; Brown, W. Colloid Polym. Sci. 1996, 274, 1033. (e) Candau, S. J . In Theoretical Challenges in the Dynamics of Complex Fluids; McLeish, T., Ed.; Kluwer Academic Publishers: Dordrecht, 1997.

(6) Lieser, G.; Oda, M.; Miteva, T.; Meisel, A.; Nothofer, H. G.; Scherf, U.; Neher, D. Macromol ecules 2000, 33, 4490.

(7) Friend, R. H.; et al. Nature 1999, 397, 121. Nothofer, H. G.; et al. Nature 2000, 405, 661.

(8) Berne, J. B.; Pecora, R. Dynamic Light Scattering; WileyInterscience Publications: New York, 1976.

(9) Provencher, S. W. Macromol. Chem. 1979, 180, 201.

(10) Grell, M.; Bradley, D. D. C.; Chamberlain, M.; Inbasekarn, M.; Woo, E. P.; Soliman, M. Acta Polym. 1998, 49, 439.

(11) Klaerner, G.; Miller, R. D. Macromolecules 1998, 31, 2007.

(12) Pecora, R. J . Chem. Phys. 1968, 48, 4126. Rallison, J. M.; Leal L. G. J . Chem. Phys. 1981, 74, 4819. Kubota, K.; Urabe, H.; Tominaja, Y.; Fugima, S. Macromolecules 1984, 17, 2096. Claire, K.; Pecora, R. J . Phys. Chem. 1997, 101, 746.

(13) Yoshizaki, T.; Yamakawa, H. J . Chem. Phys. 1980, 72, 57.

(14) Doi, M.; Edwards, S. F. The Theory of Polymer Dynamics; Oxford University Press: New York, 1986. 